\title{
A Access of MBFD, Percentage Util, Absolute Capacity and BSP VM Placement Algorithm on Cloud Datacenter
}

\author{
Sreenivasa B.L, S Sathyanarayana
}

\begin{abstract}
The datacenter control admission is expanding hurriedly because of the versatility and dynamic provisioning of benefits. The debasement in like manner generally execution, effect of surroundings are the vital inconveniences in datacenter for the blast in power utilization. The power benefited from by method for unused host device to be turned off, through combining the virtual gadget powerfully to keep control in datacenter. on this paper we have conversely 4 specific virtual machine situation set of rules for the quality utilization, amount of VM movement, SLA infringement (SLAV), in general execution debasement in view of Migration (PDM), SLA infringement Time in accordance with vigorous host (SLATAH) and power SLA infringement (ESV). to assess the arrangement of guidelines we have utilized the CloudSim recreation toolbox and true works of art burden lines of planet lab VMs for our examination.
\end{abstract}

catchphrases:-control utilization, VM relocation, SLA infringement (SLAV), execution debasement in light of Migration (PDM), SLA infringement Time steady with energetic host (SLATAH) and power SLA infringement (ESV).

\section{CREATION}

Distributed computing with the help of web it lets in the client to get section to and control the insights without building up the framework asset at client's place. character get passage to their assets remotely and need never again dread about security and insurance inconveniences. the rule highlight of distributed computing is valuable asset sharing among different clients by the strategy for virtualization [1].

With virtualization, severa computerized gadget is united to a solitary host machine in datacenter, in this way dealing with elite and execution. considerably less host apparatus usage finishes in less power utilization, bounty significantly less zone utilized and diminished immense sort of electric added substances; prompting unpracticed registering [2].

The outstanding burdens are dynamic in nature, the host contraption burden keeps on different, bringing about generally speaking execution debasement if there should be an occurrence of CPU overutilization or vitality admission development in various case. in this way stay movement of advanced device are finished in the cloud datacenter through solidifying the virtual machine i.e., moving the virtual device from one host to each unique host to meet the different remaining burden and bringing down the measure of energetic host machines [3].

there are various explanations behind the blast in vitality utilization in datacenter, anyway in that wasteful usage of

Revised Manuscript Received on August 19, 2019.

Sreenivasa B.L, Research Scholar, Bharathiar University, Assistan Professor, Department of MCA, AIMIT College, St Aloysius College, Mangalore, Karnataka.India

Dr.S Sathyanarayana, Asst. Professor, First Grade Women's College, Mysore, Karnataka.India host gadget is one reason chargeable for the blast in power consumption by method for method for the host device.

For power proficiency of the Cloud datacenters there can be basically 4 head areas that need to be advanced [5]. these are as per the following:

$\square$ discover the underneath stacked host in datacenter and found them in drowsing mode with the guide of relocating the majority of the VMs to uncommon dynamic host (underneath burden discovery).

$\square$ decide the host that is over-burden. Move some VMs from the analyzed over-burden host to various host inside the datacenters while saving QoS (Overload recognition).

$\square$ determine the $\mathrm{VM}(\mathrm{s})$ that should be moved (VM inclination).

$\square$ vicinity the picked VMs on unique energetic or reactivated has (VM arrangement).

in this paper we can be centering at the fourth detail that is VM arrangement. We interestingly four exact VM position calculations for the various run of the mill execution metric through way of utilizing CloudSim reproduction toolbox. For our assessment we have utilized the source code, design records, and impacts which can be openly to be had from http://sourceforge.net $/ \mathrm{p} / \mathrm{vm}$ alloc/evaluation/[13]. The remainder of the paper is sorted out as pursues. segment 2 clarifies VM position set of guidelines. fragment three insights concerning trial arrangement. portion four clarifies surrenders give final product of four VM arrangement calculation on particular execution measurements. at long last portion 5 closes the paper giving future way.

\section{VM SITUATION ALGORITHMS}

A. modified acceptable solid lessening set of principles $(M B F D)$

The Beloglazov et al proposed an altered fine suit bringing down calculation (MBFD) for dynamic VM solidification dependent on an assessment of verifiable data from the valuable asset use by means of VMs. the creator Beloglazov et al broke down various host over-burden and VM decision set of rules and inferred that the charming outcomes are done if near to Regression an over-burden location calculation joined with the base movement Time (MMT) VM decision inclusion are utilized. The calculation lessens vitality admission, decline the SLA infringement and the quantity of VM movements [8].

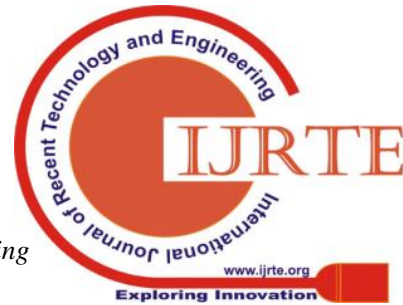




\section{B. PercentageUtil and AbsoluteCapacity set of approaches}

The author Shi et al in correlation and assessed numerous VM situation calculations. the author Shi et al proposed a whole number direct programming variant for the greatest satisfying VM position with the targets of limiting the dynamic PMs and scope of relocations for PM combinations. the creator Shi et al structured and assessed some of receptacle pressing calculations utilizing particular arranging systems to choose leading which one is best at combining VMs in data focus allowing unused PMs to be have turned out to be off. in addition, severa methodologies had been better than \% dynamic VM demands inside the most extreme green way. everything about various canister pressing calculations provided a couple of advancement inside the amount of PMs which can be fueled off due to combination, notwithstanding when the wide sort of VM movements needed for this union was also taken underneath consideration. PercentageUtil and AbsoluteCapacity are the 2 set of guidelines which completed appropriately. In every case, the PMs are dealt with customary with the given measurement (utilization and usefulness, separately), and after that the calculation iteratively attempts to free the littlest PM with the valuable asset of attempting to relocate its VMs to the greatest PMs [6].

\section{Backward Speculative Placement (BSP)}

The Calcavecchia et al proposed the BSP calculation. Which tests the relocation of all VMs of the most stacked PM to every single other Pm. From the potential movements, it chooses the one fundamentally dependent on authentic remaining task at hand measurements, impacts in the littlest peril of call for disappointment, utilizing a scoring system called "name for risk" [7]

\section{CHECKING OUT CONDITION \& RESULTS}

\section{A. experiment Setup}

On this investigate, we have in correlation four calculations in CloudSim 3.0.three and broke down the presentation of four extraordinary VM position calculation. we have considered 800 heterogeneous real hubs, half of which may be HP ProLiant G4 and the rest are HP ProLiant G5 servers. power utilization is determined dependent on HP ProLiant G4 and HP ProLiant G5 CPU use and vitality admission that is spoken to in work area 1 [4]. the ones servers are allocated with 1860MIPS (Million training in venture with second) and 2660 MIPS for each center of G4 and G5 servers individually. arrange transmission capacity is contemplated as $1 \mathrm{~GB} / \mathrm{s}$. The VMs which have been made have been single focus. VM had been of 4 sorts, for instance, over the top CPU Medium occasion (2500 MIPS, zero.80 five GB); more prominent monstrous model (2000 MIPS, 3.75 GB); Small occurrence (a thousand MIPS, 1.7 GB); and Micro example (500 MIPS, $613 \mathrm{MB}$ ).

in this work we have utilized real global depictions burden records that is given from CoMon task, a checking foundation for PlanetLab [9]. This records is gathered from more prominent than thousand VMs of various servers which can be situated in 500 stand-out areas. The remaining burden is specialist of an IaaS cloud condition by and large with Amazon EC2, wherein VMs are made and overseen by methods for the utilization of a few unprejudiced clients. table 2 gives the day smart VM extend for this records. those genuine worldwide follows incorporate VM usage data in every 5-min c program languageperiod.

\section{B. overall execution Metrics}

To have the option to test the general by and large execution of set of rules in general execution we remember the accompanying measurements to evaluate [14].

- preferred power admission - general vitality utilization is depicted as the whole of solidarity benefited from through the physical resources of a data focus as a result of programming remaining burdens.

- SLA infringement - SLA infringement are the infringement in the organization level understanding. On the off chance that the necessities of the customers aren't met, at that point we get SLA infringement.

The 2 metric for estimating the SLA infringement in anIaaS surroundings are:

SLA infringement time steady with dynamic host (SLATAH) is the total of the considerable number of infringement for every one of the hosts even as apportioned MIPS is substantially less than asked MIPS.

execution debasement as a result of movements (PDM), showing the normal in general execution corruption, with respect to have potential, because of relocations

- Type of VM movement - as fast as the over-burden or underneath-stacked server watched the VMs get settled on for relocation. Relocation uses switches and switches it additionally eat more noteworthy power and basic movement decreases amazing of administration expected to diminish standard sort of relocations in the datacenter.

- ESV - It's a blend of vitality admission and SLA infringement. it might be treated as regular size. On the off chance that the result of vitality admission and SLA infringement is decline, it way that the methodology diminishes control admission and making a horrendous parcel substantially less SLA infringement.

Table 1 Power consumption by the selected servers at different load levels in Watts

\begin{tabular}{|l|l|l|l|l|l|l|}
\hline \multirow{2}{*}{$\begin{array}{l}\text { Machine } \\
\text { Type }\end{array}$} & \multicolumn{6}{|c|}{ Power Consumption Based on CPU utilization } \\
\cline { 2 - 7 } & $0 \%$ & $20 \%$ & $40 \%$ & $60 \%$ & $80 \%$ & $100 \%$ \\
\hline HP G4 (Watt) & 86 & 92.6 & 99 & 106 & 112 & 117 \\
\hline HP G5 (Watt) & 93.7 & 101 & 110 & 121 & 129 & 135 \\
\hline
\end{tabular}

Table 2.Selected trace-based workloads.

\begin{tabular}{|l|l|l|l|}
\hline Workload & No. of VMs & Workload & No. of VMs \\
\hline 20110303 & 1052 & 20110403 & 1463 \\
\hline 20110306 & 898 & 20110409 & 1358 \\
\hline 20110309 & 1061 & 20110411 & 1233 \\
\hline 20110322 & 1516 & 20110412 & 1054 \\
\hline 20110325 & 1078 & 20110420 & 1033 \\
\hline
\end{tabular}




\section{Simulation Results and Analysis}

In our experiment, the use of CloudSim, we've compared four special VM placement algorithms. we've got used nearby regression (LR) as overload detection set of rules and maximum Correlation (MC) VM choice coverage. The desk 3 suggests the end result of our test.

Table 3. Comparison of four different VM placement Algorithms

\begin{tabular}{|c|c|c|c|c|}
\hline & MBFD & $\begin{array}{l}\text { Percent } \\
\text { ageUtil }\end{array}$ & $\begin{array}{l}\text { AbsoluteC } \\
\text { apacity }\end{array}$ & BSP \\
\hline $\begin{array}{l}\text { Energy } \\
\text { Consumption } \\
(\mathrm{kWh})\end{array}$ & 151.37 & 117.85 & 115.72 & 126.80 \\
\hline $\begin{array}{l}\text { VM } \\
\text { Migration }\end{array}$ & 22833 & 8143 & 9939 & 12964 \\
\hline SLATAH & $7.07 \%$ & $5.00 \%$ & $3.86 \%$ & $4.11 \%$ \\
\hline PDM & $0.10 \%$ & $0.04 \%$ & $0.04 \%$ & $0.06 \%$ \\
\hline $\begin{array}{l}\text { SLAV } \\
\left(10^{-2}\right)\end{array}$ & $\begin{array}{l}0.0068 \\
5\end{array}$ & $\begin{array}{l}0.0020 \\
6\end{array}$ & 0.00162 & $\begin{array}{l}0.0023 \\
1\end{array}$ \\
\hline $\begin{array}{l}\text { ESV } \\
\left(10^{-2}\right)\end{array}$ & 1.03 & 0.24 & 0.18 & 0.29 \\
\hline $\begin{array}{l}\text { Average } \\
\text { SLA } \\
\text { Violation }\end{array}$ & $9.44 \%$ & $9.51 \%$ & $9.87 \%$ & $10.30 \%$ \\
\hline $\begin{array}{l}\text { Number of } \\
\text { Host } \\
\text { Shutdowns }\end{array}$ & 4088 & 890 & 987 & 1342 \\
\hline
\end{tabular}

- Discourse on execution of seven VM scenario calculation with admire to every measurement are given beneath.

- Standard vitality admission - the main role of this evaluation is to comfy the jogging of VM arrangement set of requirements concerning pleasant admission. discern 1 demonstrates the very last product of the energy admission of 4 precise calculations. The MINLP set of ideas devours negligible vitality while contrasted with shutting calculations.

- SLA infringement - SLA infringement is one of the key markers of QoS. determine 2 indicates the SLA Violation of four unmistakable calculations. The outcome having low SLA infringement guarantees the favored QoS. The MINLP set of pointers having insignificant SLAV whilst contrasted with specific calculations.

- SLATAH - determine 3 demonstrates the very last made of four distinct calculations. The MINLP calculation have insignificant SLA infringement because of over-burden.

- PDM - discern four suggests the result of four explicit calculations. The MINLP calculation have least SLA infringement because of $\mathrm{VM}$ relocation.

- extensive assortment of VM relocation - appreciably less number of VM movement way powerful solidification, much less guests in cloud set up and much less SLA infringement for VM relocation. parent five demonstrates the aftereffect of 4 unique calculations. The MINLP set of standards have less
VM relocation in evaluation with shutting calculations.

- ESV - parent 6 shows the very last made from ESV for four selective calculations. The MINLP set of requirements have negligible ESV very last product. ESV is negligible manner spares energy and indistinguishable time SLA infringement is overseen.

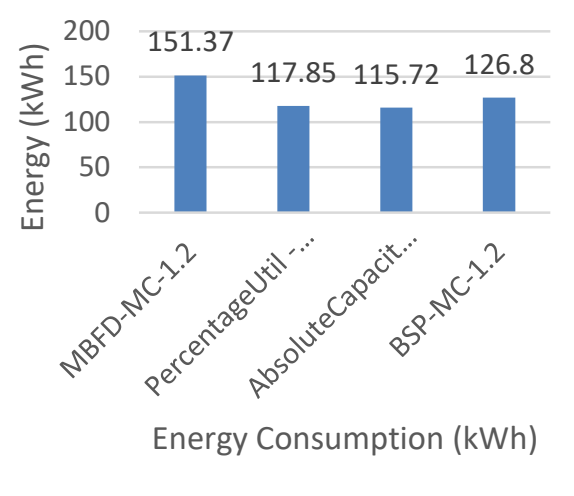

Figure 1 Energy Consumption Chart

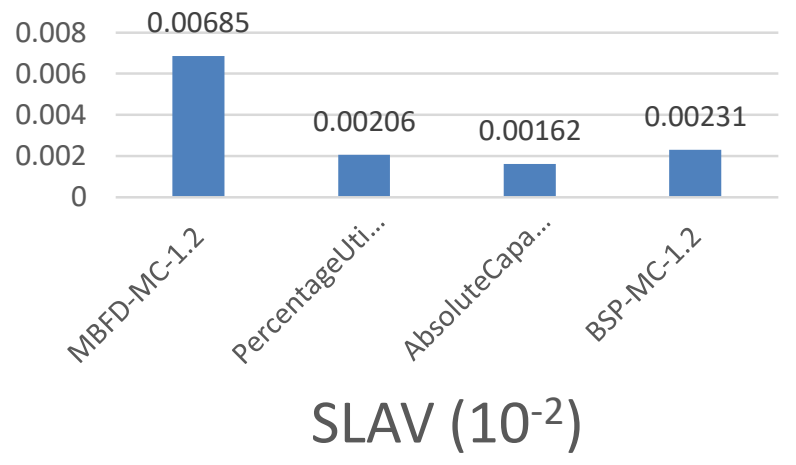

Figure 2 SLA Violation Chart

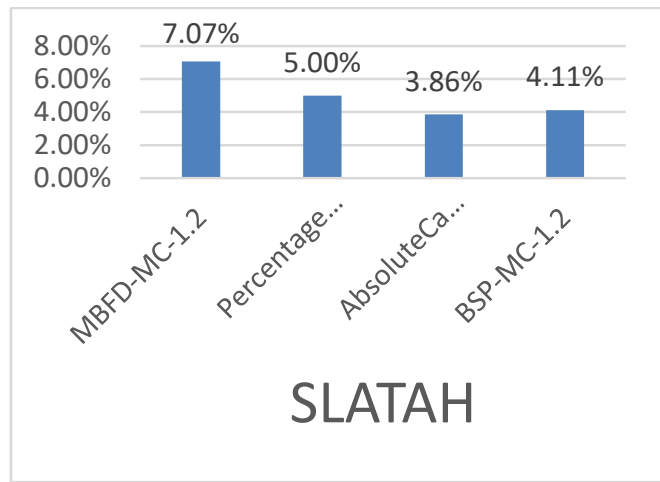

Figure 3 SLATAH Chart 


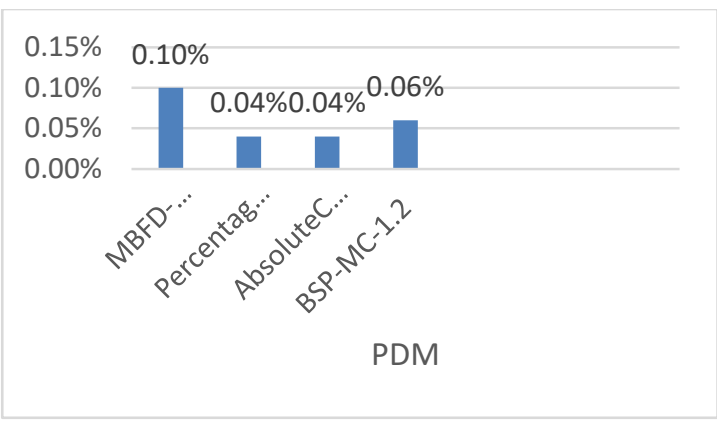

Figure 4 PDM Chart

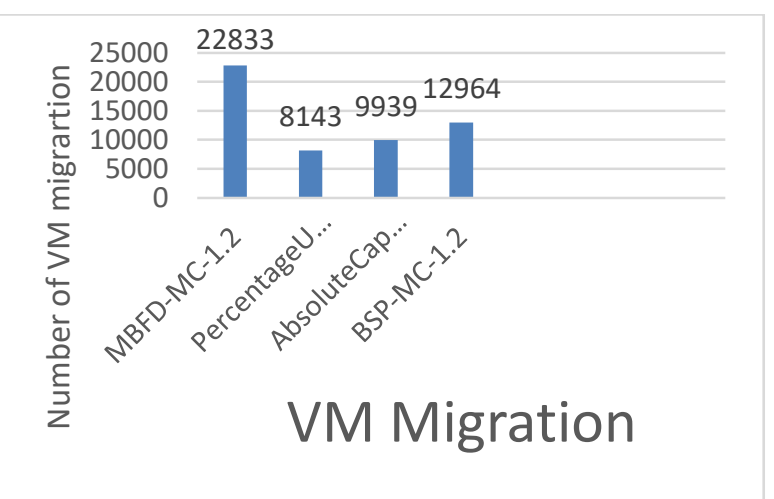

Figure 5 VM Migration Chart

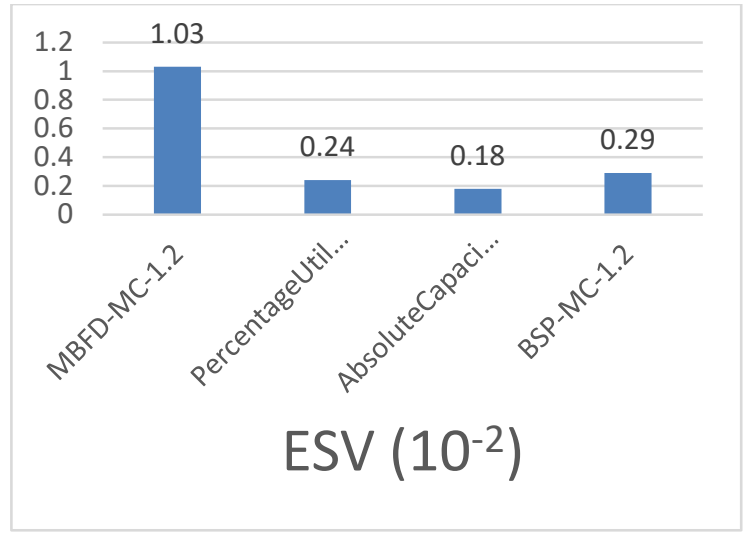

Figure 6 ESV Chart

\section{CONCLUSION}

The energy admission is the finest enterprise inside the datacenter resulting from increment widespread for registering assets. the difficulty is an method to regulate the earnings by using techniques for bringing down the energy utilization along SLA. on this paper we've got looked at 4 top notch automatic framework function calculation for the first-rate admission, scope of VM relocation, SLA infringement (SLAV), execution debasement because of Migration (PDM), SLA infringement Time consistent with enthusiastic host (SLATAH) and power SLA infringement (ESV). to assess the arrangement of standards we've utilized the CloudSim reenactment toolbox and real global works of art burden lines of planet lab VMs for our check.

From our investigation we comprehended that MINLP calculation devours less power, least SLAV, SLATAH, PDM, VM relocation and ESV.

As a predetermination bearing, this work shows us to analyze the calculation on diverse closing task handy traces,
CPU heap of VMs, VM length, PM heterogeneity, PM power traits. This empowers to realize how the calculation plays at the formerly mentioned measurements.

\section{REFERENCES}

1. Jay H. Sheth, Prof. Krunal N. Vaghela, "specialised diagram on stay virtual machine Migration techniques for Eucalyptus Cloud", Jay H. Sheth Int. mag of Engineering examination and bundles, ISSN : 2248-9622, Vol. five, issue three, ( factor - 2) March 2015, pp.50-52.

2. Server Virtualization: a piece nearer to price productivity and endeavor Agility, Avanade edge, 2009.

3. Dabiah Ahmed Alboaneen1, BernardiPranggono and HuagloryTianfield," manage mindful virtual contraption Consolidation for Cloud facts Centers2014 IEEE/ACM 7th worldwide display on programming and Cloud Computing.

4. Beloglazov A, (2013) $\mathrm{PhD}$ Thesis: "strength productive administration of virtual Machines in data communities for Cloud Computing". hyperlink: http://beloglazov.info/thesis.pdf. Gotten to 04 Jul 2016.

5. MohammadAlaulHaqueMonilandRashedur M. Rahman, VM solidification strategy dependent on heuristics fluffy reason, and motion oversee, mag of allotted computing: Advances, contraption and bundles, DOI 10.1186/s13677-016-0059-7.

6. L. Shi, J. Furlong, and R. Wang. Experimental appraisal of vector container pressing calculations for electricity efficient insights focuses. In IEEE Symposium on pc frameworks and Communications, pages 9-15, 2013.

7. Nicolo Maria Calcavecchia, OferBiran, ErezHadad, and YosefMoatti. VM role approaches for cloud conditions. In IEEE fifth popular show on Cloud Computing (CLOUD), pages 852859, 2012.

8. Anton Beloglazov and RajkumarBuyya, chief online Deterministic calculations and versatile heuristics for power and execution inexperienced unique union of virtual machines in cloud facts places of work, Concurrency Computat.: Pract. Exper. 2012; 24:1397-1420, published on line in Wiley InterScience (www.interscience.wiley.com). DOI: 10.1002/cpe.1867.

9. Park KS, Pai VS (2006) CoMon: a normally adaptable checking system for planet-Lab. ACM SIGOPS working Syst Rev 40(1):sixty 5-seventy four.

10. KyoungSoo Park and Vivek S. Pai. CoMon: an basically adaptable checking tool for Planet-Lab. ACM SIGOPS operating systems evaluation, forty(1):sixty 5seventy 4, 2006.

11. Larry Peterson, Andy Bavier, Marc E. Fiuczynski, and Steve Muir. contemplatesbuildingPlanetLab. In court instances of the seventh Symposium on operating structures format and Imple-mentation, pages 351366, 2006.

12. Charles Reiss, Alexey Tumanov, Gregory R Ganger, Randy H Katz, and Michael A Kozuch.Heterogeneity and dynamicity of mists at scale: Google follow examination. In protests of the3rd ACM Symposium on Cloud Computing, 2012.

13. SiqiShen, Vincent van Beek, and AlexandruIosup. Measurable portrayal of businesscriticalworkloads facilitated in cloud datacenters. In fifteenth IEEE/ACM worldwide Symposiumon Cluster, Cloud and Grid Computing, pages 465474, 2015.

14. ArockiaRanjini and ArunSahayadhas, A differentiation see of various superior framework combination calculations in cloud datacenter, ARPN magazine of Engineering and did Sciences, VOL. 12, NO. 1, JANUARY 2017, ISSN 1819-6608.

15. Zoltan Adam Mann and Mate Szabo, this is the exceptional set of principles for advanced system state of affairs streamlining, simultaneousness and calculation practice and recognize, degree 29, trouble 10, e4083. 
16. D. G. doLago, E. R. M. Madeira, and L. F. Bittencourt. vitality cognizant virtual framework booking on mists the utilization of enthusiastic cooling manipulate and DVFS. In persevering with In strategies of the ninth global Workshop on Middleware for Grids, Clouds and e-technology, 2011.

17. M. Guazzone, C. Anglano, and M. Canonico. Abusing VM motion for the robotized electricity and execution the executives of unpracticed distributed computing frameworks. In first international Workshop on quality talented server farms, pages 8190 . Springer, 2012.

18. Mohammed Rashid Chowdhury, Mohammad Raihan Mahmud, and Rashedur M. Rahman. take a gander at and execution evaluation of diverse VM situation structures. In sixteenth IEEE/ACIS worldwide display on programming mission Engineering, manufactured Intelligence, Networking and Parallel/assigned Computing (SNPD). IEEE, 2015.

19. L. Shi, J. Furlong, and R. Wang. Observational assessment of vector receptacle pressing calculations for first-rate inexperienced statistics workplaces. In IEEE Symposium on pc frameworks and Communications, pages 9-15, 2013.

20. Nicolo Maria Calcavecchia, OferBiran, ErezHadad, and YosefMoatti. VM association structures for cloud occasions. In IEEE fifth average show on Cloud Computing (CLOUD), pages 852859, 2012.

21. Minas L, Ellison B. energy execution for information age: how to decrease electricity admission in Servers and statistics places of work. Intel Press, 2009.

22. Fan X, Weber WD, Barroso 1. a..energy provisioning for a distribution middle predicted computer. protests of the 34th Annual worldwide Symposium on laptop shape (ISCA 2007), ACM ny, massive apple, the united states, 2007; thirteen-23. 